\title{
Laser ionization of ${ }^{124} \mathrm{Ag}$ and its decay to levels of ${ }^{124} \mathrm{Cd}$
}

\author{
T. Kautzsch, ${ }^{1}$ W. B. Walters, ${ }^{2}$ V. N. Fedoseyev, ${ }^{3}$ Y. Jading, ${ }^{1,4}$ A. Jokinen, ${ }^{4}$ I. Klöckl, ${ }^{1}$ K.-L. Kratz, ${ }^{1}$ \\ V. I. Mishin, ${ }^{3}$ H. L. Ravn, ${ }^{4}$ P. Van Duppen, ${ }^{5}$ A. Wöhr, ${ }^{5}$ and ISOLDE Collaboration \\ ${ }^{1}$ Institut für Kernchemie, Universität Mainz, D-55099 Mainz, Germany \\ ${ }^{2}$ Department of Chemistry, University of Maryland, College Park, Maryland 20742 \\ ${ }^{3}$ Institute of Spectroscopy, Russian Academy of Sciences, RU-142092 Troitzk, Russia \\ ${ }^{4}$ CERN, CH-1211 Genève 23, Switzerland \\ ${ }^{5}$ Instituut voor Kern-en Stralingsfysica, University of Leuven, B-3001 Leuven, Belgium
}

(Received 12 August 1996)

\begin{abstract}
Gamma-rays following the decay of the neutron-rich isotope ${ }^{124} \mathrm{Ag}$ have been studied at the ISOLDE facility using a chemically selective laser ion source. Excited structures beyond the first $2^{+}$level in ${ }^{124} \mathrm{Cd}$ have been identified for the first time, and are discussed in terms of a recent anharmonic vibration model. [S0556-2813(96)50612-6]
\end{abstract}

PACS number(s): 21.60.Ev, 23.20.Lv, 25.40.Sc, 27.60.+j

The structure and decay of neutron-rich ${ }_{45} \mathrm{Rh}$ to ${ }_{50} \mathrm{Sn}$ isotopes play a crucial role in the development of a description of the $A \simeq 120-130$ region of the astrophysical $r$ process by which about half of the nuclear species in nature heavier than iron are synthesized. Stable nuclides produced by this nucleosynthesis process show enhanced solar-system $r$ abundances $\left(N_{r, \odot}\right)$ near mass $A \simeq 80,130$, and $195[1,2]$ that have been attributed to the nuclear-structure properties of their $r$ process progenitors lying on and near the closed neutron shells at $N=50,82$, and 126 . In particular, the identification and study of neutron-magic nuclides, such as ${ }^{79} \mathrm{Cu}_{50}$, ${ }^{80} \mathrm{Zn}_{50}$, and ${ }^{130} \mathrm{Cd}_{82}$, has made possible quantitative calculations of these $N_{r} \odot$ enhancements [3].

A number of extensive calculations for the structure and decay properties of the nuclides with $110 \leqslant A \leqslant 125$ were unable to reproduce the observed $N_{r, \odot}$ pattern [4,5]. However, recent efforts that utilize a weakening of the strength of the $N=82$ closed shell below $Z=50$ have proved more successful [6].

Looking for experimental fingerprints of this effect, we have embarked on a series of spectroscopic measurements in order to determine the structure and decay properties of neutron-rich nuclides with $Z<50$ and $N \simeq 82$. The goal of these studies at CERN-ISOLDE is to investigate structure and decay properties of the neutron-rich $\mathrm{Ag}$ and $\mathrm{Cd}$ isotopes by taking advantage of the chemical selectivity of laser ionization [7] to enhance $\mathrm{Ag}$ production relative to the isobaric background usually obtained with conventional plasma ion sources. The new data for the neutron-rich Ag nuclides and their $\mathrm{Cd}$ daughters can then be compared to theoretical models in which the strength of the $N=82$ closed shell becomes a variable.

Extensive data for the structure of the even-even $\mathrm{Cd} \mathrm{nu-}$ clides has been provided by in-beam spectroscopy, radioactive decay studies and, more recently, by fission-fragment $\gamma$-ray spectroscopy [8]. However, these approaches have only been able to provide information as far as ${ }^{122} \mathrm{Cd}$. The only literature data for ${ }^{124} \mathrm{Ag}$ has been a 540(80) $\mathrm{ms}$ half-life derived from $\beta$-delayed neutron ( $\beta \mathrm{dn})$ measurements [9], and the identification of a single $\gamma$ line at $613 \mathrm{keV}$ that exhibited a 170(30) ms half-life [10]. In both studies, the Ag was produced in a conventional on-line mass separator setup.

In a recent note [11], we reported the use of a laser ion source (LIS) at the new PS-Booster ISOLDE facility to enhance the ionization of $\mathrm{Ag}$ relative to other isobaric activities that permitted the first $\beta \mathrm{dn}$ measurements of the half-lives of ${ }^{125-127} \mathrm{Ag}$. The half-life of ${ }^{124} \mathrm{Ag}$ was remeasured and found to be $172(5) \mathrm{ms}$.

In the present experiment, an improved version of the LIS described in detail in Ref. [7] has been used. This laser setup installed at the ISOLDE facility consists of three copper vapor tubes, one of them working as an oscillator and two as amplifiers at a pulse repetition rate of $10 \mathrm{kHz}\left(P_{\text {total }}=55 \mathrm{~W}\right)$, and two dye lasers equipped with microprocessor controlling of the wavelengths. Ultraviolet radiation was generated by frequency doubling with a nonlinear barium-beta-borate (BBO) crystal. The laser beams were focused over a distance of $20 \mathrm{~m}$ through a window in the separator magnet into a hole $\left(d_{\text {hole }}=2 \mathrm{~mm}\right)$ of the ion source.

In its current configuration, $\mathrm{Ag}$ nuclides are produced at ISOLDE through the spallation of uranium in a thick (44 $\mathrm{g} / \mathrm{cm}^{2}$ ) $\mathrm{UC}_{2}-\mathrm{C}$ target using a $1 \mathrm{GeV}$ beam of protons. The proton beam is produced in $2.4 \mu \mathrm{s}$ bursts, separated by multiples of $1.2 \mathrm{~s}$. These sudden short bombardments stimulate a fast release of the reaction products from the target material giving rise to moderate decay losses of short-lived isotopes. After diffusing towards a heated niobium cavity, to which the laser light is focused, the Ag atoms are ionized and are accelerated and mass separated. In addition to laser ionization of $\mathrm{Ag}$, surface ionization of elements with low ionization potential, such as In and Cs, cannot be avoided in the hot cavity. In fact, for $A=124$ the actual spallation production of the two In isomers is about 30 times stronger than that of ${ }^{124} \mathrm{Ag}$, and $\mathrm{Cs}$ is produced approximately 200 times stronger. However, by choosing optimum temperature conditions (ionizer temperature $2000 \mathrm{~K}$ ) surface ionization of In could be reduced to $0.7 \%$. Note that due to its high ionization potential, the $\mathrm{Cd}$ isotopes are not ionized at all. For the same conditions, the LIS efficiency for $\mathrm{Ag}$ was determined to $10.8 \%$. Hence, when operating the ion source without laser, we only observe the decay of surface-ionized In and Cs. On 


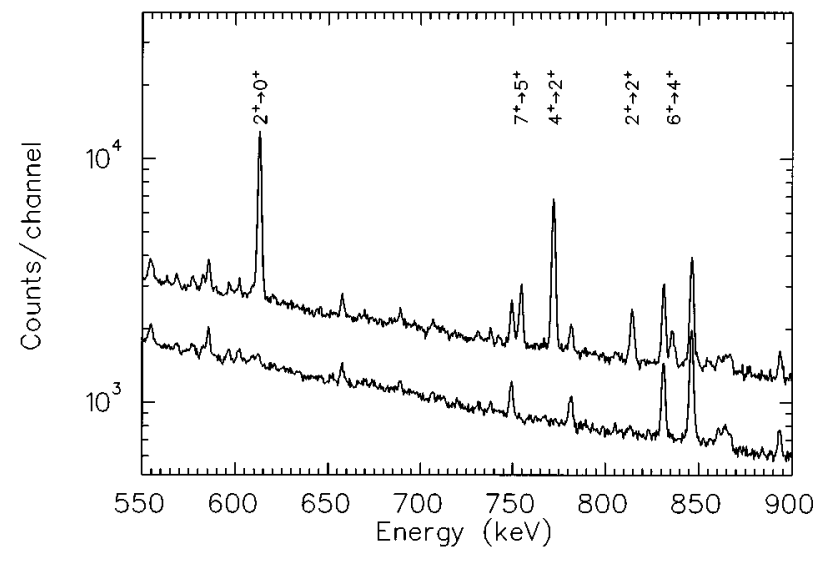

FIG. 1. Partial $\gamma$-ray spectra for two ion-source conditions. With the lasers off, only $\gamma$ lines of surface ionized In and Cs isotopes are observed (lower spectrum). With the lasers on, $\gamma$ lines of Ag can clearly be observed in addition (upper spectrum).

the other hand, when operating the ion source with the laser on, the $\mathrm{Ag}$ yield is enhanced to the point that $\mathrm{Ag}$ and its daughter $\mathrm{Cd}$ are observed with $\beta$-intensities approximately equal to those of the surface ionized In nuclides (see Fig. 1).

The ion beam was implanted on a tape which was moved periodically to minimize buildup of daughter and granddaughter activities and to remove long-lived isobaric contaminations. Two large $\mathrm{HpGe}$ detectors were placed at $90^{\circ}$ relative to each other and were at $3-$ and $8-\mathrm{cm}$ distances from the collection point. Due to severe electronic noise produced by the $10 \mathrm{kHz}$ pulsing of the lasers, the $\gamma \gamma$-coincidence measurements were strongly disturbed. Therefore, $\gamma$-singles data were collected in multispectrum mode in several 100-ms intervals, which were started at different times after the implantation of the beam. Hence, the assignment of $\gamma$ lines was mainly performed by comparing laser-on/laser-off spectra, and by analysis of the $\gamma$-ray half-lives.

In Fig. 1, we show two spectra of $\gamma$ rays in the energy range from $550 \mathrm{keV}$ to $900 \mathrm{keV}$, accumulated for a 200-ms period starting $40 \mathrm{~ms}$ after closing the beam gate, with the laser on, and also with the laser off. In Table I are listed the energies and intensities of the $\gamma$ rays that were found both to show a half-life of approximately $170 \mathrm{~ms}$ and to be absent in the spectra with the laser off.

In Fig. 2, we show a proposed level scheme that incorporates a number of the more intense $\gamma$ transitions. The levels below $2 \mathrm{MeV}$ are supported either by the intensity balance or by identification of two transitions from each level. The levels above $2 \mathrm{MeV}$ whose energies are shown in parentheses reflect the systematics for the lighter even-even $\mathrm{Cd}$ nuclides that are shown in Fig. 3. Two other possible levels that decay by two transitions are listed in Table I and enclosed in parentheses, but not shown in Fig. 2.

Several features of these structures and decay are of importance. The decay of ${ }^{124} \mathrm{Ag}$ to levels in ${ }^{124} \mathrm{Cd}$ is quite similar to that proposed by Zamfir et al. for the decay of the one neutron-pair lighter isotope ${ }^{122} \mathrm{Ag}$ to levels in ${ }^{122} \mathrm{Cd}$ [12]. In their study, two levels were assigned spin and parity $0^{+}$on the basis of angular correlation measurements. At the same time, the $\gamma$ rays identified as the $8^{+}$to $6^{+}$transition in ${ }^{122} \mathrm{Cd}$ in two different fission-gamma studies were also ob-
TABLE I. Energies, intensities, and placements of $\gamma$ rays observed following the decay of $172-\mathrm{ms}{ }^{124} \mathrm{Ag}$ to levels in ${ }^{124} \mathrm{Cd}$. The placements shown in brackets are not included in the level scheme shown in Fig. 2.

\begin{tabular}{|c|c|c|c|}
\hline Energy (keV) & Intensity & from level & to level \\
\hline 297.9 & 5.0 & & \\
\hline 301.1 & 5.3 & 1729 & 1428 \\
\hline 461.1 & 43.1 & 1846 & 1386 \\
\hline 534.2 & 6.6 & 1963 & 1428 \\
\hline 538.8 & 17.6 & 2385 & 1846 \\
\hline 612.9 & 100.0 & 613 & 0 \\
\hline 616.4 & 1.6 & & \\
\hline 620.2 & 2.3 & 2841 & 2221 \\
\hline 742.2 & 2.7 & & \\
\hline 754.6 & 15.0 & [2718] & 1963 \\
\hline 772.1 & 59.4 & 1385 & 613 \\
\hline 814.5 & 11.4 & 1428 & 613 \\
\hline 835.7 & 8.7 & 2221 & 1386 \\
\hline 876.2 & 1.4 & 3097 & 2221 \\
\hline 924.5 & 2.5 & [2771] & 1846 \\
\hline 1193.6 & 1.3 & & \\
\hline 1312.1 & 3.0 & & \\
\hline 1332.2 & 6.4 & [2718] & 1385 \\
\hline 1428.2 & 2.7 & 1428 & 0 \\
\hline 1729.4 & 2.1 & 1729 & 0 \\
\hline 1912.7 & 3.2 & & \\
\hline 1963.4 & 2.5 & 1963 & 0 \\
\hline 2158.3 & 1.5 & {$[2771]$} & 613 \\
\hline 2453.8 & 4.8 & & \\
\hline 2458.8 & 1.7 & & \\
\hline 2514.2 & 0.7 & & \\
\hline
\end{tabular}

served $[8,13]$. These data supported the presence of two $\beta$ isomers in ${ }^{122} \mathrm{Ag}$, similar to its isotone ${ }^{124} \mathrm{In}$ which has two isomers with half-lives of $3.2 \mathrm{~s}$ and $2.4 \mathrm{~s}$, and spins and parities tentatively assigned as $3^{+}$and $8^{-}$, respectively [14]. During our earlier Ag experiment [11], we remeasured the half-life of delayed neutrons at $A=122$ and obtained only a single component with 520(14) ms, in agreement with literature values. Isomers with indistinguishable half-lives and tentatively assigned spins and parities of $3^{+}$and $8^{-}$have also been reported for ${ }^{126} \mathrm{In}(1.6 \mathrm{~s})$ and ${ }^{128} \mathrm{In}(0.9 \mathrm{~s})$ [14].

With our considerably improved $\mathrm{Ag}$ enhancements, it was possible to reduce the uncertainty in the half-life of ${ }^{124} \mathrm{Ag}$ in the $\beta \mathrm{dn}$ measurements and now deduce a value of 172(5) ms with no evidence for a second component in the 500-ms range [9]. Within the given uncertainties, this $\beta$ dn value does not significantly differ from the 160(9) ms half-life value we obtain for the intense $613-\mathrm{keV} 2^{+}$to $0^{+}$transition, nor from the 166(20) ms and 185(71) ms half-lives that we obtain for the weaker 461- and 814-keV transitions. These latter transitions would reflect enhanced population from the high- and low-spin $\mathrm{Ag}$ isomers, respectively.

The spread in spin population in the decay of ${ }^{124} \mathrm{Ag}$ to levels of ${ }^{124} \mathrm{Cd}$, appears comparable to that observed for ${ }^{122} \mathrm{Ag}$ decay to levels of ${ }^{122} \mathrm{Cd}$, although we have not established any $0^{+}$levels. The unplaced $\gamma$ rays in the $2 \mathrm{MeV}$ to $2.5 \mathrm{MeV}$ range are likely to be transitions to the ground state 


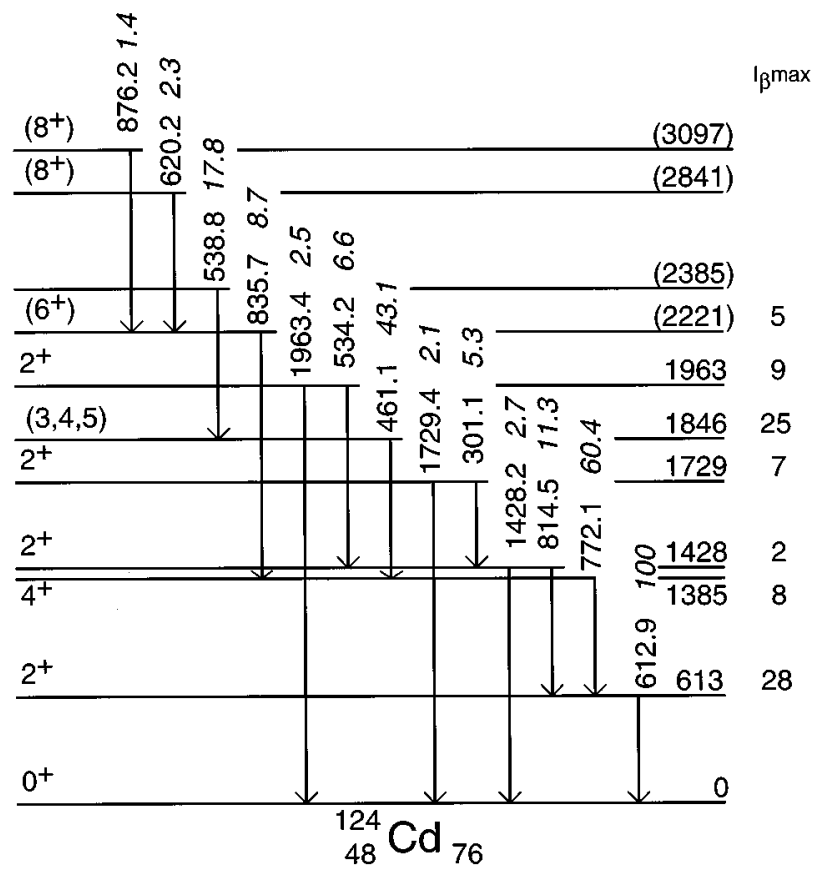

FIG. 2. Levels in ${ }^{124} \mathrm{Cd}$ populated by the decay of the ${ }^{124} \mathrm{Ag}$ ground state and a possible isomer produced in the proton spallation of an uranium target with a $1-\mathrm{GeV}$ proton beam.

or $2_{1}^{+}$state coming from levels that would be populated in decay of a low-spin isomer. At the same time, the considerable number of $\gamma$ rays in the $500 \mathrm{keV}$ to $1500 \mathrm{keV}$ range would appear to originate in higher-spin levels cascading to the yrast levels as the nucleus undergoes deexcitation. Thus, it is likely that the level scheme of ${ }^{124} \mathrm{Cd}$ we present originates from the decay of a low-spin isomer, probably $2^{+}$or $3^{+}$, and a high-spin isomer, probably $7^{-}$or $8^{-}$. Either of these isomers have relatively similar half-lives as is found for the ${ }^{126} \mathrm{In}$ isotone and suggested by the time behavior of the 461- and 814-keV transitions, or-in the case of different half-lives-one of the isomers has an extremely low $\beta \mathrm{dn}$ branch $\left(P_{n}\right)$ as only one component was found in the neutron time spectrum. And indeed, our QRPA calculations using the code of Möller and Randrup [15] predict half-lives of $103 \mathrm{~ms}$ for the $\left[\pi g_{9 / 2} ; \nu d_{3 / 2}\right]$ low-spin isomer and $133 \mathrm{~ms}$ for the $\left[\pi g_{9 / 2} ; \nu h_{11 / 2}\right]$ high-spin isomer, however with identical $P_{n}$ values of $1.1 \%$. Within this shell-model approach, we have no possibility to form a $\beta$ dn-emitting $500 \mathrm{~ms}$ 2-quasiparticle isomer in ${ }^{124} \mathrm{Ag}$ [9]. When, furthermore, assuming equal production of both isomers in $1-\mathrm{GeV}$ spallation, we must conclude that they are indistinguishable from their gross $\beta$-decay properties $T_{1 / 2}$ and $P_{n}$.

These new data for ${ }^{124} \mathrm{Cd}$ now permit some insight into the changes in collective structure in the heavy $\mathrm{Cd}$ nuclides as the $N=82$ closed shell is approached. Structures for ${ }^{118-}{ }^{124} \mathrm{Cd}$ nuclides are shown in Fig. 3. It is useful to recall that ${ }^{118} \mathrm{Cd}$ has been considered the outstanding example of a nearly pure vibrational nuclide, one that also showed some levels from particle-hole intruder states that reach a minimum in energy at $N=66$ (mid shell) $[16,17]$. For both ${ }^{118} \mathrm{Cd}$ and ${ }^{120} \mathrm{Cd}$, the low-energy structure is characterized by a nearly degenerate vibrational $0^{+}, 2^{+}, 4^{+}$triplet. In moving from ${ }^{118} \mathrm{Cd}$ to ${ }^{124} \mathrm{Cd}$, six-neutron holes have been filled, most likely in the $h_{11 / 2}$ orbitals. Hence, the collective structure of ${ }^{124} \mathrm{Cd}$ should be governed by the interactions between the two $g_{9 / 2}$ proton holes, and the six remaining neutron holes, divided among the $d_{3 / 2}, s_{1 / 2}$, and $h_{11 / 2}$ orbitals. Because the high-spin orbitals are both over half full, the interaction should be a strong hole-hole interaction, and relatively symmetrical vibrational structure should dominate. We note that the other valence orbitals, the $d_{3 / 2}$ and $s_{1 / 2}$ neutron orbitals, and $p_{1 / 2}$ proton orbitals can only play a limited role in forming collective structures. Indeed, what is observed is a narrowing of the $4^{+}-2^{+}$gap, and a drift upward in the position of the second $0^{+}$state.

Casten, Zamfir, and Brenner have shown that for many nuclides the analysis of collectivity can be carried out using a model for anharmonic vibrational nuclides, and, that for nuclides where the $E_{4} / E_{2}$ ratio lies between 2.05 and 3.15, $E_{4^{+}}$is approximately equal to $2 E_{2^{+}}+\epsilon_{4}$ [18]. In Fig. 3, the $E_{4}+E_{2}+$ ratio is shown in brackets for all of the Te and $\mathrm{Cd}$ nuclides, and $\epsilon_{4}$ is shown in parentheses. Rather universal values have been found for $\epsilon_{4}$ that depend on $Z$. For nuclides below $Z=50$, that value is $190 \pm 24 \mathrm{keV}$. Zamfir et al. ana-

$10^{+} 4277$

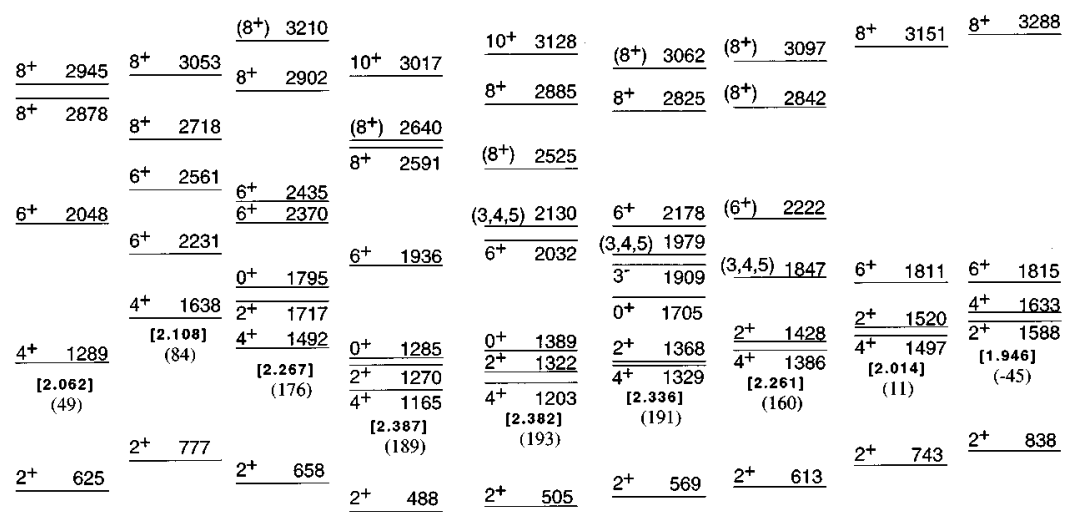

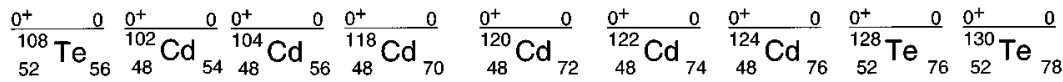

FIG. 3. Structure of the even-even $\mathrm{Cd}$ and $\mathrm{Te}$ nuclides. The $8^{+}$and $10^{+}$levels shown in ${ }^{118-122} \mathrm{Cd}$ nucleides have been observed in fission-fragment $\gamma$-ray coincidence studies. The other possible $8^{+}$levels (those with spin in parentheses) as well as the levels shown with spin $(3,4,5)$ have been observed in Ag decay studies. The numbers in brackets just below the $4^{+}$levels are the energy ratio $E_{4^{+}} / E_{2^{+}}$. The numbers in parentheses below the $4^{+}$levels are the $\epsilon_{4}$ values. 
lyzed the structure of ${ }^{122} \mathrm{Cd}$ in this means, and were able to indicate that the $6^{+}$level at $2178 \mathrm{keV}$ is a good candidate for the $6^{+}$vibrational level, and that the $8^{+}$level at $3062 \mathrm{keV}$ could be a candidate for the $8^{+}$vibrational level [5]. For comparison, we have also shown the structure for ${ }^{104} \mathrm{Cd}_{56}$ the even-even $\mathrm{Cd}$ nucleus with six neutrons beyond the $N=50$ closed shell. Both the $E_{4^{+}} / E_{2+}$ and $\epsilon_{4}$ are seen to be quite comparable to those values for the heavy $\mathrm{Cd}$ nuclides.

In Fig. 3, we have also shown the structure of the sixneutron particle and hole Te nuclides, ${ }^{108} \mathrm{Te}$ and ${ }^{128} \mathrm{Te}$, along with their $E_{4}+E_{2}$ ratio and $\epsilon_{4}$. While they differ somewhat from the $\mathrm{Cd}$ nuclides, the values for both the six-particle and six-hole nuclides are close to each other and consistent with the anharmonic vibrator picture.

We have also included in Fig. 3 the even-even Te nuclide, ${ }^{130} \mathrm{Te}$ with only four neutron holes in the $N=82$ closed neutron shell, and the even-even $\mathrm{Cd}$ nuclide, ${ }^{102} \mathrm{Cd}$ which has only four neutrons beyond the $N=50$ closed neutron shell. In both cases, significant departures from both the $E_{4} / E_{2}+$ ratio and $\epsilon_{4}$ value are found when compared with the adjacent nuclide, and in both cases, the values are lower.

Hence, these new data reported for ${ }^{124} \mathrm{Cd}$ in this paper are fully consistent with the notion that the $\mathrm{Cd}$ nuclides from six-particle ${ }^{104} \mathrm{Cd}$ through six-hole ${ }^{124} \mathrm{Cd}$, as well as their isotonic Te nuclides, show what might be termed "consistent vibrational structure" and, that the shift away from collective structure begins for both $\mathrm{Cd}$ and Te nuclides when either the holes or particles are less than six.

We noted at the outset that one approach to nuclear structure and decay in the heavy $\mathrm{Cd}$ nuclides that could account for the observed $r$-process abundances included a weakened shell structure for the $N=82$ closed neutron shell below $Z=50$. Therefore, it will be the structure of ${ }^{126} \mathrm{Cd}_{78}$ where the first experimental indication of weakened shell strength could be found. That is, the degree to which the $E_{4^{+}} / E_{2^{+}}$ ratio and $\epsilon_{4}$ value are found to shift toward lower values should be a test of the degree to which the $N=82$ shell strength is comparable to both the $N=82$ shell strength above $Z=50$ and the $N=50$ shell strength in the light $\mathrm{Cd}$ nuclides. These same laser-enhanced ionization techniques, when combined with high resolution mass separation, should make investigation of the decay of the heavier, even-mass Ag decay possible and bring observation of the structure of even-even $\mathrm{Cd}$ nuclides as far as "closed-shell", ${ }^{130} \mathrm{Cd}_{82}$ within reach.

This work was supported by the German BMBF (06 MZ 476), DFG (436 RUS 17/31/95), and by the Russian Foundation for Fundamental Research (93-02-14282).
[1] E.M. Burbidge, G.R. Burbidge, W.A. Fowler, and F. Hoyle, Rev. Mod. Phys. 29, 547 (1957).

[2] F. Käppeler, H. Beer, and K. Wisshak, Rep. Prog. Phys. 52, 945 (1989).

[3] K.-L. Kratz, J.-P. Bitzouet, F.-K. Thielemann, P. Möller, and B. Pfeiffer, Astrophys. J. 403, 216 (1993).

[4] F.-K. Thielemann, K.-L. Kratz, B. Pfeiffer, T. Rauscher, L. v. Wormer, and M.C. Wiescher, Nucl. Phys. A570, 329c (1994).

[5] K.-L. Kratz, in Nuclei in the Cosmos III, edited by M. Busso et al., AIP Conf. Proc. No. 327 (AIP, New York, 1995), p. 113 , and references therein.

[6] B. Chen, J. Dobaczewski, K.-L. Kratz, K. Langanke, B. Pfeiffer, F.-K. Thielemann, and P. Vogel, Phys. Lett. B 355, 37 (1995).

[7] V.I. Mishin, V.N. Fedoseyev, H.-J. Kluge, V.S. Letokhov, H.L. Ravn, F. Scheerer, Y. Shirakabe, S. Sundell, O. Tengblad, and the ISOLDE Collaboration, Nucl. Instrum. Methods Phys. Res. B 73, 550 (1993).

[8] J.H. Hamilton, A.V. Ramayya, S.J. Zhu, G.M. Ter-Akopian,
Yu.Ts. Oganessian, J.D. Cole, J.O. Rasmussen, and M.A. Stoyer, Prog. Part. Nucl. Phys. 35, 635 (1995).

[9] P.L. Reeder, R.A. Warner, and R.L. Gill, Phys. Rev. C 27, 3002 (1983).

[10] J.C. Hill, F.K. Wohn, Z. Berant, R.L. Gill, R.E. Chrien, C. Chung, and A. Aprahamian, Phys. Rev. C 29, 1078 (1984).

[11] V.N. Fedoseyev et al., Z. Phys. C 353, 9 (1995).

[12] N.V. Zamfir, R.L. Gill, D.S. Brenner, R.F. Casten, and A. Wolf, Phys. Rev. C 51, 98 (1995).

[13] J.L. Durrell, in The Spectroscopy of Heavy Nuclei 1989, edited by J.F. Sharpey-Schafer and L.D. Skouras, IOP Conference Series 105 (Institute of Physics, Bristol, 1990), p. 307.

[14] B. Fogelberg and P. Carle, Nucl. Phys. A323, 205 (1979).

[15] P. Möller and J. Randrup, Nucl. Phys. A514, 1 (1990).

[16] A. Aprahamian, D.S. Brenner, R.F. Casten, R.L. Gill, and A. Pietrowski, Phys. Rev. Lett. 59, 535 (1987).

[17] H. Mach et al., Phys. Rev. Lett. 63, 143 (1989).

[18] R.F. Casten, N.V. Zamfir, and D.S. Brenner, Phys. Rev. Lett. 71, 227 (1993). 